\title{
Activation of Astrocytes in Vitro by Macrophages Polarized with Keratin Biomaterial Treatment
}

\author{
Bailey V. Fearing1, Mark E. Van Dyke ${ }^{2}$ \\ ${ }^{1}$ Department of Orthopaedic Surgery, Wake Forest University School of Medicine, Winston Salem, NC, USA \\ ${ }^{2}$ Department of Biomedical Engineering and Mechanics, Virginia Polytechnic Institute and State University, \\ Blacksburg, VA, USA \\ Email: mvandyk5@vt.edu
}

Received 5 December 2015; accepted 1 March 2016; published 4 March 2016

Copyright (C) 2016 by authors and Scientific Research Publishing Inc.

This work is licensed under the Creative Commons Attribution International License (CC BY). http://creativecommons.org/licenses/by/4.0/

(c) (i) Open Access

\section{Abstract}

Reactive astrocytes contribute to glial scarring by rapid proliferation and up-regulation of glial fibrillary acidic protein (GFAP) expression and production of chondroitin sulfate proteoglycans (CSPGs). CSPGs play a crucial role in formation of the glial scar, which takes over the lesion site following spinal cord injury (SCI). This process corresponds to the inflammatory response of macrophages, which polarize toward a dominant pro-inflammatory M1 phenotype following SCI. The M1 phenotype is known to release various cytotoxic compounds that exacerbate the glial scar, which in turn impedes tissue regeneration. Recent studies have shown that anti-inflammatory M2 macrophages play a role in allowing neurite extensions to occur, even across inhibitory substrates, and can lessen the degree of secondary damage. Based on earlier results demonstrating that keratin biomaterials may polarize macrophages toward an anti-inflammatory M2 phenotype, we test the hypothesis that these polarized macrophages will have the potential to indirectly effect astrogliosis. Using an in vitro model of reactive astrogliosis, macrophage-conditioned media from cells that have been cultured with soluble keratin for 24 hours or 7 days appears to decrease reactivity and associates CSPG production. These results are statistically similar to the control M2 macrophage conditioned media. A comparable collagen-conditioned macrophage media does not resolve astrocyte reactivity, while control $\mathrm{M} 1$ macrophage conditioned media results in an increase in GFAP expression. These data suggest keratin-derived macrophages are more functionally similar to M2 macrophages and that keratin treatment may aid in limiting secondary inflammatorymediated damage.

\section{Keywords}

Keratin, Macrophage, Astrocyte, Glial Scar, Inflammation, Spinal Cord Injury, CSPGs 


\section{Introduction}

One of the more pronounced late-stage outcomes following a spinal cord injury (SCI) is the formation of an astroglial scar, a reactive process that involves glial cells, mostly astrocytes, accumulating and enveloping the injury site [1]-[3]. In its early stages, the glial scar is beneficially important in attempting to stabilize the defective blood spinal cord barrier (BSB) and minimizing the spread of cellular damage [3]-[5]. However, its unintended effect is to create a barrier to regenerate axons through physical and chemical interactions. A dense network of glial cells and their processes physically block advancement of the growth cone, while inhibitory molecules and cytotoxic compounds held within the extracellular matrix (ECM) of the scar present chemical barriers to axon growth [6]. Chondroitin sulphate proteoglycans (CSPGs) are structural biomolecules that make up a major class of inhibitory substrates found within the glial scar but a normal component in the central nervous system (CNS). Their role in the CNS appears to be in controlling neuronal differentiation by guiding axons and restricting growth to only those proper targets, as well as preventing migration of neural crest cells, both crucial steps in neuronal development [7]-[9]. CSPGs have also been shown to regulate neuronal plasticity by forming perineuronal nets around synapses [10] [11].

Of the CSPG-producing glial cells, astrocytes possess a more dominant role because of the range of CSPGs produced and their close association with the glial scar. Following injury in the CNS, astrocytes undergo a process known as reactive astrogliosis. The resulting reactive astrocytes contribute considerably to the glial scar and rapidly up-regulate production of CSPGs [12] [13]. Astrocytes produce a range of CSPGs known to be inhibitory, specifically aggrecan, brevican, neurocan, versican, and phosphacan [3] [14]-[17]. It is well established that the inflammatory nature of the SCI lesion site contributes greatly to secondary damage, including the infiltration and activation of macrophages and perpetuating reactive astrogliosis. Astrocytes express cell surface receptors for inflammatory cytokines like interleukin (IL)-6, IL-1 $\beta$, interferon- $\gamma$ (IFN $\gamma$ ), tumor necrosis factor- $\alpha$ (TNF $\alpha$ ), and transforming growth factor $\beta$ (TGF $\beta$ ) and it has been shown that treating astrocytes with such inflammatory cytokines causes them to become reactive [18]. One of the major sources of such cytokines results from classical activation of macrophages to an M1 phenotype. M1 macrophages dominate the SCI lesion site and are known to be cytotoxic and inhibit neuron growth. M2 macrophages, conversely, promote repair and allow for greater axon growth across inhibitory CSPG substrates, but significantly decline in number by one week post-injury [19]. It is known that the characteristically high presence of reactive astrocytes is associated with the rapid up-regulation of TGF $\beta$ following CNS injury [20] [21]. Such an increase in TGF $\beta$ stimulates M2 macrophages to become "deactivated" and subsequently produce higher levels of TGF $\beta$ themselves. The overproduction of TGF $\beta$ by macrophages encourages an increase in ECM proteins and a decrease in matrix turnover, which consequently leads to structural and functional damage, as well as contributes to the fibrotic scar [22]. Interactions between activated macrophages and reactive astrocytes in the lesioned spinal cord are beginning to be understood but research is ongoing. It has been demonstrated that conditioned media from M2 macrophages promotes axon sprouting without concurrent neurotoxicity, unlike conditioned media from M1 macrophages [19]. Additionally, the same study shows that combining M2 macrophage-conditioned media with chondroitinase ABC (chABC) results in three to fivefold increases in axon growth across a CSPG gradient. Moreover, M2 macrophage-conditioned media is more effective at supporting and synergizing with chABC to produce such outcomes.

A new intervention being studied for SCI is keratin biomaterials. A keratin hydrogel has demonstrated efficacy in promoting greater recovery of gait and bladder functions following a rat hemisection injury [23]. It has also shown an ability to skew macrophage polarization toward a M2 phenotype and promote significant up-regulation of M2-associated cytokines like IL-10, while down-regulating M1-associated cytokines like IL-6 [24]. Therefore, we hypothesized that macrophages polarized by treatment with keratin would be capable of paracrine-mediated modulation of astrocyte behavior. In this study, we used an in vitro method to investigate the effects of keratin-treated macrophage conditioned media (K-MCM) on reactive astrocytes by quantifying their production of CSPGs following treatment.

\section{Methods and Materials}

All materials were purchased from Fisher Scientific, unless otherwise indicated, and used as received. Deionized (DI) water was obtained from an in-house generation system. 


\subsection{Preparation of Keratin Biomaterial and Coatings}

Keratin biomaterial was extracted and prepared as previously described [25] [26]. Briefly, a 2\% solution of peracetic acid was used to oxidize human hair fibers. After rinsing with DI water, soluble keratin proteins were extracted with successive treatments of tris(hydroxymethyl)aminomethane (Tris) base and DI water. The extracts were combined and following dialysis, the solution was neutralized to $\mathrm{pH} 7.4$ with $\mathrm{NaOH}$, lyophilized, and ground into a powder. A $25 \mathrm{kGy}$ dose of $\gamma$-irradiation was used to sterilize the keratin powder, which was then reconstituted in phosphate buffered saline (PBS). Keratin and type-1 collagen (BD Biosciences) solutions were diluted to a final concentration of $200 \mu \mathrm{g} / \mathrm{ml}$ and $1 \mathrm{ml}$ of corresponding solutions were added to the wells of a glass chamber slide (Nunc, Thermo Fisher Scientific). Coatings were formed by incubating glass chamber slides for 24 hours at $37^{\circ} \mathrm{C}$, followed by removal of excess solution and rinsing with PBS prior to cell seeding.

\subsection{Macrophage Conditioned Media and Astrocyte Cell Culture}

The THP-1 human monocytic cell line was obtained from ATCC (Manassas, VA) and maintained in RPMI 1640 (Gibco Life Technologies) containing $4.5 \mathrm{~g} / \mathrm{L}$ D-glucose, 10mM HEPES, and $1 \mathrm{mM}$ sodium pyruvate and was supplemented with $10 \%$ fetal bovine serum (FBS), $1 \%$ penicillin/streptomycin (P/S), and $0.05 \mathrm{mM} 2$ 2-mercaptoethanol. THP-1-derived macrophages (TDM) were generated by plating $1 \times 10^{6}$ cells in six-well plates (Becton Dickinson) and treating with $5 \mathrm{ng} / \mathrm{ml}$ phorbol myristate acetate (PMA; Sigma) for 48 hours at $37^{\circ} \mathrm{C}$ in $5 \%$ $\mathrm{CO}_{2}$. TDMs were washed with PBS, trypsinized (0.25\% trypsin/0.1\% EDTA: HyClone), pelleted, and seeded on respective coatings and treatment groups. Control treatments were conducted using LPS (100 ng/ml; Sigma) and human recombinant (hr) IFN $\gamma$ (20 ng/ml; Sigma) to create M1 macrophages. M2 macrophages were produced by treating with hrIL-4 (20 ng/ml; Sigma). TDMs cultured in the presence of growth media alone were considered M0 macrophages. Media was collected after 24 hours and centrifuged for 10 minutes at $1000 \times \mathrm{g}$ to remove any particulate matter. Media was changed every three days for the 7 day conditioned media and it should be noted that the media collected had been exposed to 7 day old cultured cells for 24 hours. Media conditioned from 24 hours will hereinafter be referred to as Treatment A and media conditioned from 7 days will be referred to as Treatment B. Samples were stored at $-80^{\circ} \mathrm{C}$ until use.

Primary human astrocytes were purchased from ScienCell at passage one (fetal brain donor). Cells were maintained on $2 \mu \mathrm{g} / \mathrm{cm}^{2}$ poly-l-lysine coated T-75 flasks in complete astrocyte medium (AM) (ScienCell) with $5.5 \mathrm{mM}$ glucose in $5 \% \mathrm{CO}_{2}$ at $37^{\circ} \mathrm{C}$. Medium was changed every other day until cell confluence reached $90 \%$ when cells were sub-cultured. Fetal astrocytes were expanded and maintained to reach desired cell density prior to experimental use, approximately 3 weeks and up to passage 4 .

\subsection{In vitro Reactive Astrogliosis Assay}

Human astrocytes were plated on glass chamber slides (Nunc, Thermo Fisher Scientific) at $5000 \mathrm{cells} / \mathrm{cm}^{2}$ in complete astrocyte media. Cells were treated with $1 \mu \mathrm{g} / \mathrm{ml}$ TGF- $\beta 1$ (R \& D Systems) for 5 days to create reactive astrocytes (RA) [20] [21] [27]. They were then washed with PBS and media was replaced with MCM or control treatment for an additional 5 days (Table 1). Media was changed every third day. MCM was chosen from two time points from a previous study [24]. Media conditioned for 24 hours and media conditioned for 7 days was collected because those time points demonstrated the least and greatest effects observed in macrophage polarization, respectively.

\subsection{Immunocytochemistry}

All stains were performed at room temperature (RT) using an optimized immunofluorescence technique. Astrocyte cultures were divided into two staining groups: triple-stained group using versican, GFAP, and aggrecan, and double-stained group with CS-56 and GFAP. For the triple-staining, astrocytes cultured on glass slides were washed with PBS and treated with $0.02 \mathrm{U}$ protease-free chABC (Sigma) for 3 hours at $37^{\circ} \mathrm{C}$ to remove glucosaminoglycan (GAG) chains from the core proteins. Cells were then fixed with $4 \%$ paraformaldehyde (PFA) for 20 minutes at RT. Non-specific staining was blocked with $10 \%$ bovine serum albumin (BSA) for 45 minutes at RT. Cultures were washed (0.1\% BSA) and the first primary antibody, versican (1:500; Novus Biologics) was added for one hour. After washing, the secondary antibody was added (AlexaFluor ${ }^{\circledR}$ 633, 1:200; Jackson Laboratories) for one hour in the dark. The second primary antibody, GFAP (1:500; Dako), was added after rinsing for 
an additional hour, followed by the secondary antibody Rhodamine $\operatorname{Red}^{\circledR}$ (1:150; Jackson Laboratories). Lastly, FITC-conjugated aggrecan antibody (1:200; Novus Biologics) was added for one hour in the dark. For the double-stained group, astrocytes were washed and fixed with 4\% PFA for 20 minutes at RT. No chABC digestion was necessary since CS-56 antibody targets the GAG portion rather than the core protein. Anti-chondroitin sulfate CS-56 (1:200; Sigma) was added for one hour at room temperature. Cells were washed and a secondary AlexaFluor ${ }^{\circledR} 488$ (Jackson Laboratories) antibody was added (1:200) for one hour in the dark. The second primary antibody, GFAP, was added for one hour in the dark, washed, and its secondary antibody Rhodamine Red ${ }^{\circledR}$ was incubated for one hour. Following the triple- and double-staining, gaskets were removed from the chamber slides and slides were mounted with ProLong ${ }^{\circledR}$ Gold Antifade mounting media (Life Technologies) and visualized using a Zeiss LSM510 inverted confocal microscope. Technical controls consisted of stained cells from each treatment group without primary antibodies (Table 2).

Table 1. Culture treatment conditions of human astrocytes; ${ }^{*}$ denotes naïve astrocytes that were not treated with TGF- $\beta 1$.

\begin{tabular}{lc}
\hline Treatment Group & Conditions \\
K-MCM A & Keratin MCM 24 hours \\
K-MCM B & Keratin MCM 7 days \\
C-MCM A & Collagen MCM 24 hours \\
C-MCM B & Collagen MCM 7 days \\
M1-MCM A & M1 (LPS/IFN $\gamma$ ) MCM 24 hours \\
M1-MCM B & M1 (LPS/IFN $\gamma$ ) MCM 7 days \\
M2-MCM A & M2 (IL-4) MCM 24 hours \\
M2-MCM B & M2 (IL-4) MCM 7 days \\
M0-MCM A & No coating MCM 24 hours \\
M0-MCM B & No coating MCM 7 days \\
RA-RPMI & Non-conditioned macrophage media \\
K-RPMI & Non-conditioned macrophage media* \\
RA-AM & Astrocyte media* \\
RPMI & Astrocyte media \\
AM & 0.001 mg/mL Keratin in non-conditioned macrophage media \\
\hline
\end{tabular}

Table 2. Details of antibodies utilized in this study.

\begin{tabular}{cccc}
\hline Antibody & Vendor & Cat. No. & Host \\
\hline Versican & Novus Biologics & 16770002 & Rabbit polyclonal \\
AlexaFluor633 & Life Technologies & A21070 & Goat anti-rabbit \\
Aggrecan-FITC & Novus Biologics & NB100-74350F & Rabbit polyclonal \\
CS-56 & Sigma & C8035 & Mouse monoclonal \\
AlexaFluor 488 & Jackson Immuno Research & Goat anti-mouse & Rabbit polyclonal \\
GFAP & Dako & 111-297-003 & Goat anti-rabbit \\
Rhodamine Red & Jackson Immuno Research &
\end{tabular}




\subsection{CSPG Quantitative Analysis}

Quantitative analysis of aggrecan, versican, CS-56, and GFAP-positive staining areas was conducted by selecting fields on each slide with $20 \times$ magnification. Nine pre-determined areas were chosen on each sample in order to avoid overlapping areas. These pre-determined fields were consistent and in the same locations on all sample slides. Regions used for quantification were as follows: 3 fields located within the top third of the slide, 3 fields in the middle third of the slide, and 3 fields on the bottom third of the slide. All areas were analyzed for relative fluorescence using ImageJ (NIH) software. Replicates of 3 for each treatment and control group were analyzed. MCM treatments A and B were compared to the corresponding M0-MCM treatments A and B values because M0-MCM represents media conditioned with a mature, but unpolarized macrophage phenotype.

\subsection{Statistical Analysis}

Relative fluorescence units (RFU) of control and MCM groups were compared using a one-way analysis of variance (ANOVA) with a Bonferroni's post-hoc test. For MCM conditions, the average mean value of M0-MCM was calculated and indicated on the graphs as a dashed line. Significant values are compared to the mean value of M0-MCM from treatment A and treatment B of each CSPG (versican, aggrecan, CS-56) marker and GFAP. All tests were completed using Prism v.5.0 (GraphPad Software Inc.) and data presented as mean \pm standard error of the mean (SEM) with $p<0.05$ being considered statistically significant.

\section{Results}

\subsection{Macrophage Media Does Not Contribute to Reactive Astrogliosis}

Naïve astrocytes cultured with macrophage media alone (RPMI) did not exhibit any changes in CSPG or GFAP expression (data not shown). When reactive astrocytes were cultured with macrophage media (RA-RPMI) there was no statistical difference in aggrecan, versican, or CS-56 compared to RA-AM (Figures 1(A)-(C)). There was also no change in overall reactivity as indicated by GFAP marker expression (Figure 1(D)). Macrophage media containing solubilized keratin (K-RPMI) demonstrated a significant decrease in aggrecan (Figure 1(B)) and GFAP (Figure 1(D)).

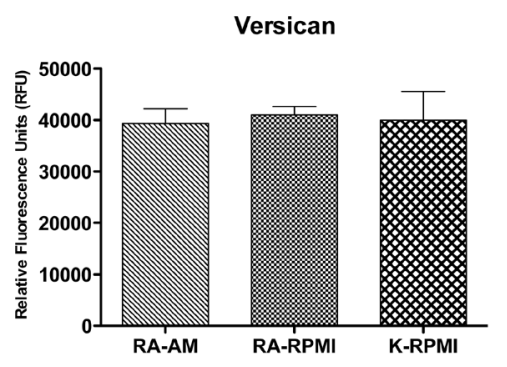

(A)

CS-56

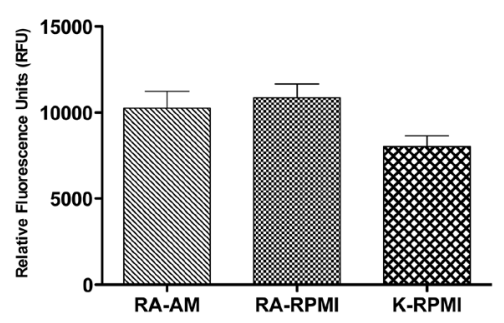

(C)

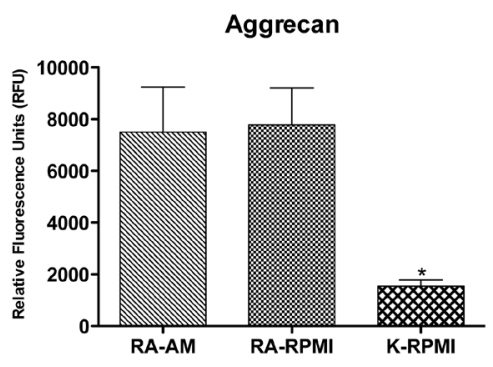

(B)

GFAP

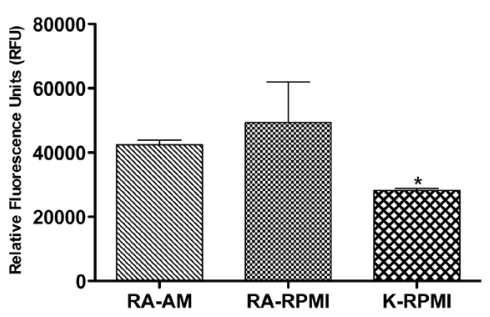

(D)

Figure 1. Control treatments show macrophage media does not influence reactive astrogliosis. There was no statistical difference in versican (A), aggrecan (B), CS-56 (C) or GFAP (D) expression in the presence of macrophage media compared to astrocyte media. There was a significant decrease in aggrecan (B) and GFAP (D) promoted by keratin solubilized in macrophage media (K-RPMI). RA-AM: reactive astrocytes-astrocyte media; RA-RPMI: reactive astrocytes-RPMI (macrophage) media; K-RPMI: keratin (solubilized)-RPMI media. " $p<0.05$, 1-way ANOVA (Bonferroni's post-hoc). 


\subsection{Keratin-Conditioned Media Treatments A and B Promote Diminished CSPG Production}

Reactive astrocytes cultured in the presence of keratin-conditioned media (K-MCM) produce significantly less versican, aggrecan, and CS-56 when compared to M0-MCM treatments A (Figures 2(A)-(C)) and B (Figures 3(A)-(C)). A similar trend was observed with M2 positive control conditioned media (M2-MCM) treatments A and B showing a significant decline in versican (Figure 2(A) and Figure 3(A)), aggrecan (Figure 2(B) and Figure 3(B)), and CS-56 (Figure 2(C) and Figure 3(B)). GFAP expression was significantly decreased with K-MCM and M2-MCM for both treatments A (Figure 2(D)) and B (Figure 3(D)) compared to M0-MCM. There was no statistical difference observed between any M2-MCM and K-MCM groups.

\subsection{Collagen-Conditioned Media Has No Effect on Reactive Astrocytes}

Reactive astrocytes cultured in the presence of collagen-conditioned macrophage media (C-MCM) showed no statistical difference in aggrecan, versican, and CS-56 for both treatments A (Figures 2(A)-(C)) and B (Figures 3(A)-(C)) compared to M0-MCM. There was also no change in GFAP expression from C-MCM treatments A (Figure 2(D)) and B (Figure 3(D)). M1-MCM treatment A resulted in a significant increase in GFAP expression (Figure 2(D)), while promoting a significant increase in CS-56 (Figure 2(C)) and no difference in versican and aggrecan production compared to M0-MCM treatment A (Figure 2(A) and Figure 2(B)). M1-MCM treatment B caused no change in versican and CS-56 expression (Figure 3(A) and Figure 3(C)) compared to that of M0-MCM, while promoting a significant increase in GFAP (Figure 3(D)) and a significant decrease in aggrecan (Figure 3(B)).

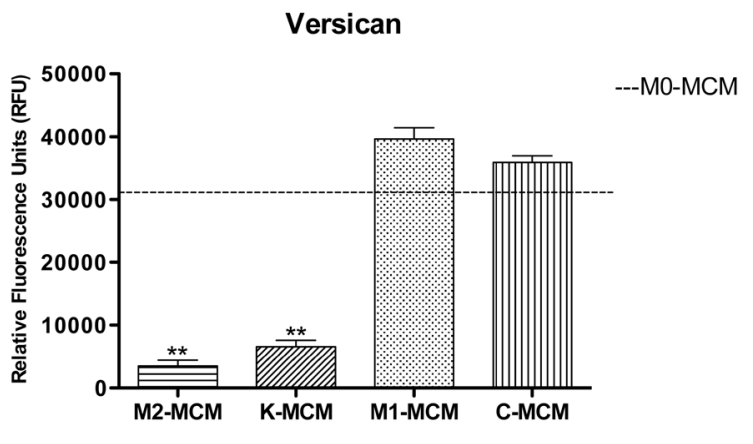

(A)

CS-56

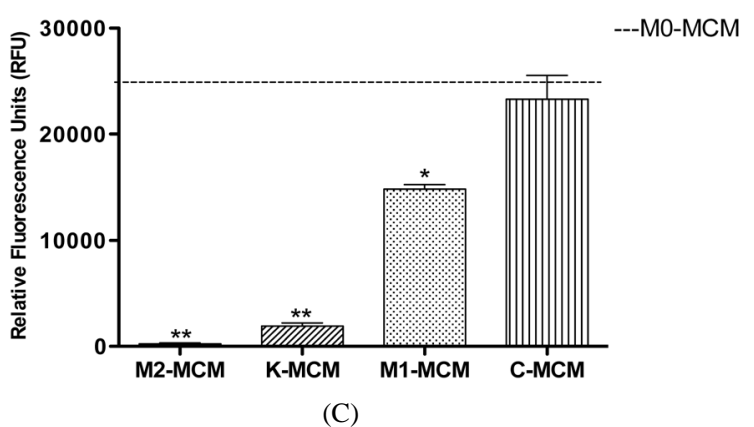

(C)

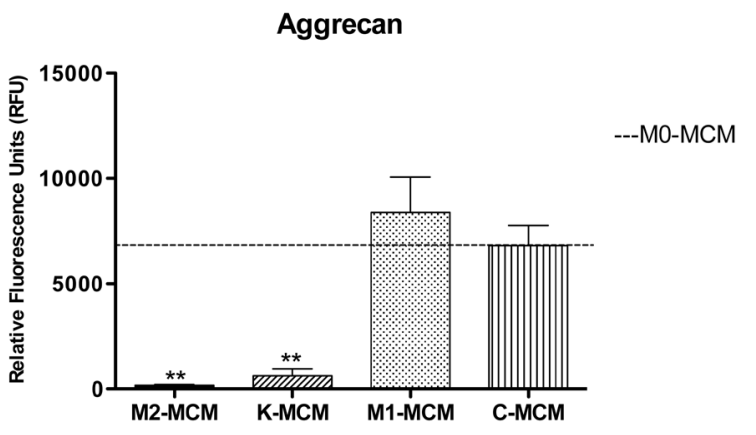

(B)

GFAP

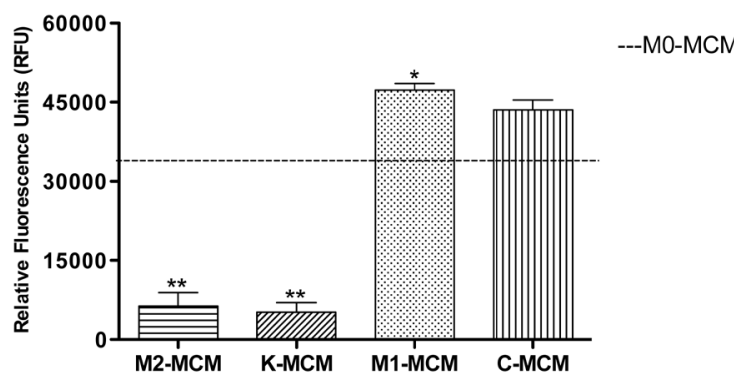

(D)

Figure 2. CSPG and GFAP expression by reactive astrocytes cultured in treatment A, macrophage conditioned medium. There is a significant decrease in versican (A); aggrecan (B); CS-56 (C); and GFAP (D) in the M2-MCM and K-MCM groups compared to the M0-MCM average mean of treatment A. There was no statistical difference between C-MCM and M0-MCM treatment A in versican (A); aggrecan (B); CS-56 (C); and GFAP (D) immunofluorescence. GFAP expression significantly increased with M1-MCM treatment A compared to M0-MCM (D), while CS-56 expression significantly decreased with M1-MCM compared to M0-MCM (C). M0-MCM average mean of Treatment A (M0-MCM Tx A mean values in RFU: versican, 30864.5; aggrecan, 6599.26; CS-56, 25048.67; GFAP, 33789.26).M1-MCM: M1-macrophage conditioned media; C-MCM: collagen-macrophage conditioned media; M2-MCM: M2-macrophage conditioned media; K-MCM: keratin-macrophage conditioned media. ${ }^{*} p<0.05,{ }^{* *} p<0.01$, 1 -way ANOVA (Bonferonni’s post-hoc). 


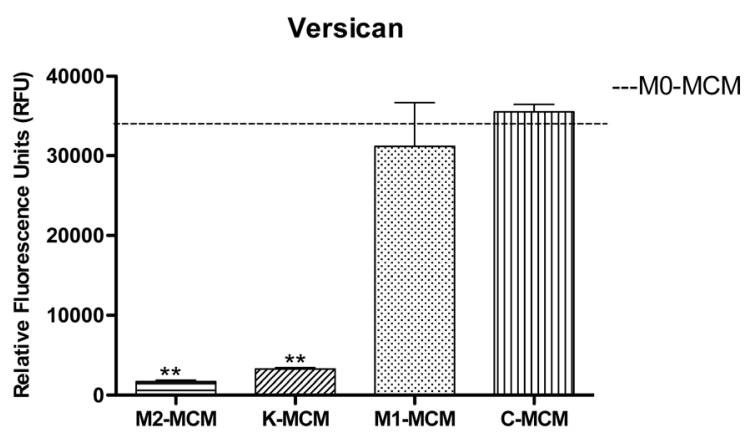

(A)

CS-56

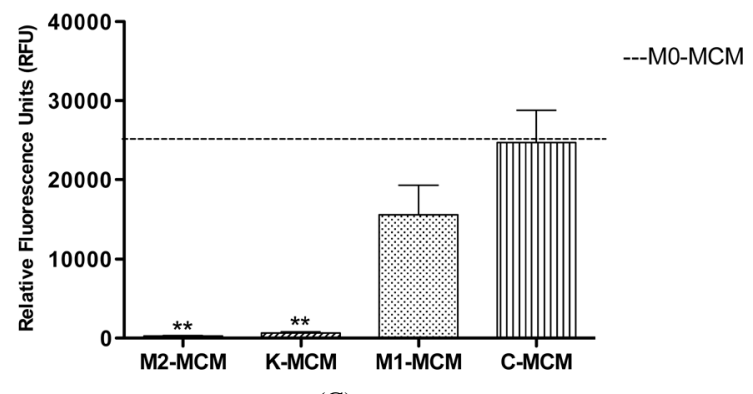

(C)

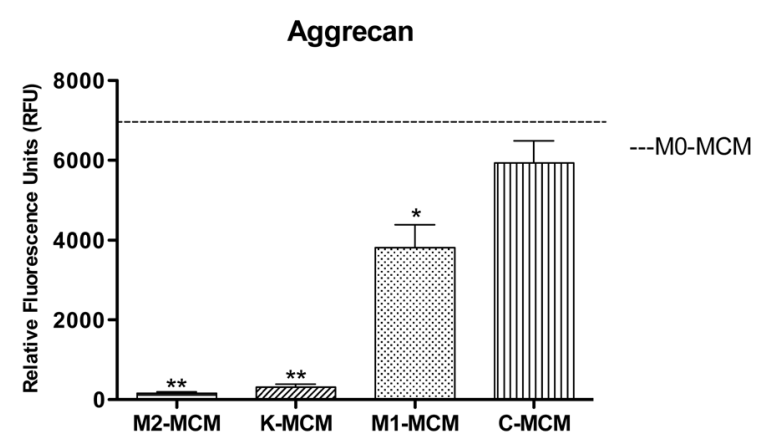

(B)

GFAP

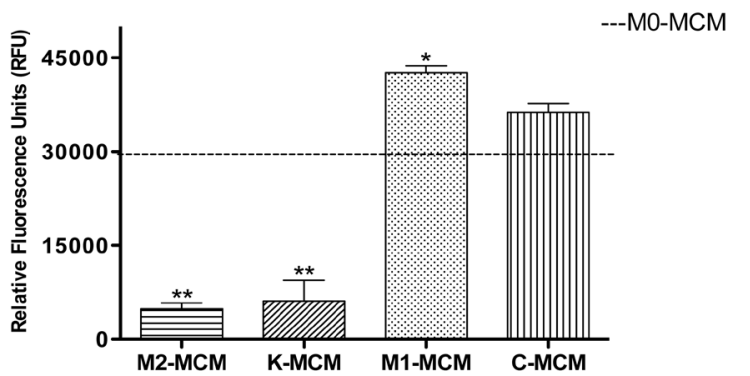

(D)

Figure 3. CSPG and GFAP expression by reactive astrocytes cultured in treatment B macrophage conditioned medium. There is a significant decrease in versican (A); aggrecan (B); CS-56 (C); and GFAP (D) in the M2-MCM and K-MCM groups compared to the M0-MCM average mean of treatment B. There was no statistical difference between C-MCM and M0-MCM treatment B in versican (A); aggrecan (B); CS-56 (C); and GFAP (D) immunofluorescence. GFAP expression significantly increased with M1-MCM treatment B compared to M0-MCM (D), while aggrecan production significantly decreased with M1-MCM compared to M0-MCM (B). -M0-MCM average mean of Treatment B (M0-MCM Tx B mean values in RFU: versican, 33947.67; aggrecan, 6974.93; CS-56, 25075.7; GFAP, 33789.26). M1-MCM: M1-macrophage conditioned media; C-MCM: collagen-macrophage conditioned media; M2-MCM: M2-macrophage conditioned media; K-MCM: keratin-macrophage conditioned media. ${ }^{*} p<0.05,{ }^{* *} p<0.01,1$-way ANOVA (Bonferonni's post-hoc).

\section{Discussion}

It is well known that a glial scar forms at the lesion site following SCI and is largely responsible for the nonpermissive environment inhibiting axon regeneration. Major components of the glial scar are reactive astrocytes and their associated CSPGs. Several studies have shown that by diminishing CSPGs, regeneration and functional outcomes improve [28]-[30]. The present study shows that media conditioned in the presence of macrophages polarized to different phenotypes have an effect on reactive astrogliosis in vitro (Figure 4 and Figure 5). This suggests that factors secreted by these macrophages can influence downstream inflammatory and repair mechanisms either positively or negatively, depending on the function of the specific phenotype (i.e. pro- or anti-inflammatory), in particular astrogliosis (Figure 2 and Figure 3). Several groups have demonstrated the relationship between activated (M1 and M2) macrophage-conditioned media on neurons, as well as that of reactive astrocytes on neurons [18] [19] [31]. However, the role of M1 and M2 macrophages in reactive astrogliosis is not as well-characterized and only recently was it demonstrated that conditioned media from M1 macrophages can cause astrocytes to become more reactive compared to conditioned media from M2 macrophages [32].

M1 and M2 macrophages release an array of cytokines and chemokines that are well established as having deleterious and growth-promoting effects (respectively) in the SCI lesion milieu. An early presence of M1 and M2 related cytokines in the lesion can rapidly increase the amount of surrounding reactive astrocytes [33], indicative of pro- and anti-inflammatory mechanisms that correlate with findings showing a mixed M1/M2 phenotype during the first week following SCI [19]. It was also shown that by later time points, especially when M1-macrophage bias occurs, that increased production of pro-inflammatory cytokines, such as IL-6, leads to more inflammatory reactive astrocytes. 


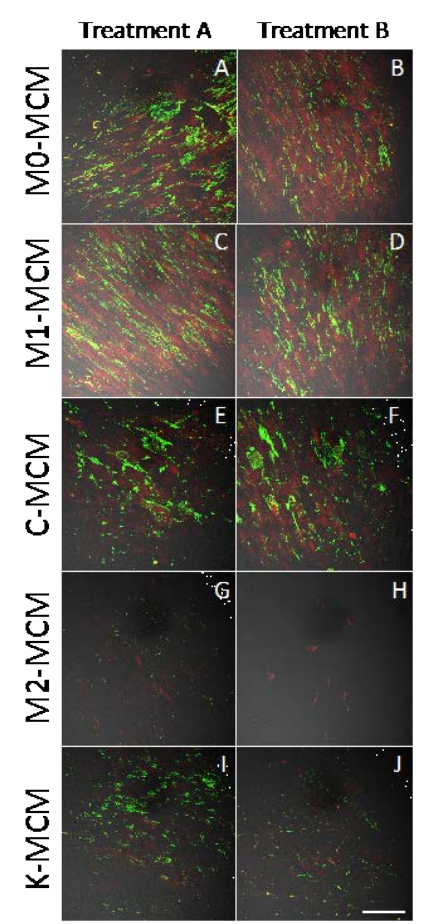

Figure 4. Immunofluorescent images of treatment groups showing CS-56 (green) and GFAP (red) expression. M0-MCM: no coating (M0)-macrophage conditioned media; M1-MCM: M1-macrophage conditioned media; C-MCM: collagen-macrophage conditioned media; M2-MCM: M2-macrophage conditioned media; K-MCM: keratin-macrophage conditioned media; Scale bar $=100 \mu \mathrm{m}$.

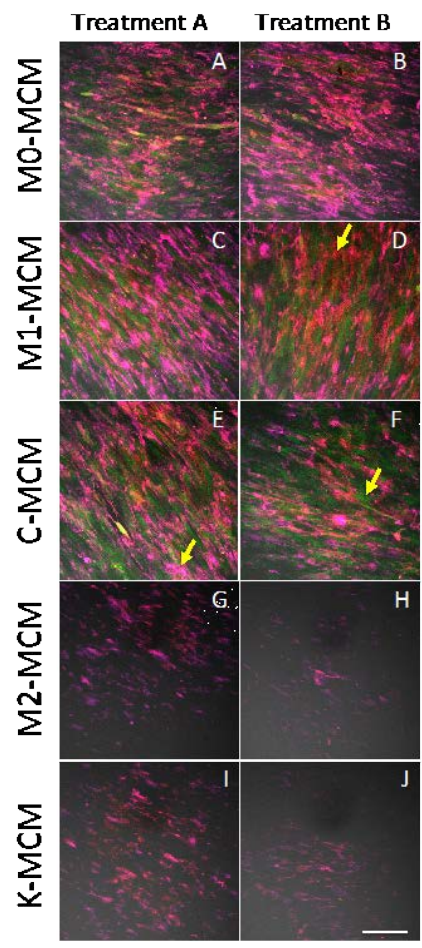

Figure 5. Immunofluorescent images of treatment groups showing versican (purple), aggrecan (green), and GFAP (red) expression. Yellow arrows indicate areas of red (D), purple (E), and green (F). M0-MCM: no coating (M0)-macrophage conditioned media; M1-MCM: M1-macrophage conditioned media; C-MCM: collagen-macrophage conditioned media; M2-MCM: M2-macrophage conditioned media; K-MCM: keratin-macrophage conditioned media; Scale bar $=100 \mu \mathrm{m}$. 
Astrocytes express cell surface receptors to common inflammatory cytokines like IL-6, IL- $1 \beta$, IFN $\gamma$, and $\mathrm{TNF} \alpha$ [19]. A previous study demonstrated a significant increase in IL-6 produced by macrophages grown on a collagen coating and those cultured in the presence of M1-driving cytokines (i.e. LPS and IFN $\gamma$ ) [24]. This finding corresponds to the current study where collagen-conditioned macrophage media produced no differences in reactive astrocyte-associated CSPG and GFAP expression (Figure 2 and Figure 3) and M1-conditioned media produced significant increases in GFAP (Figure 4 and Figure 5). However, it should be noted that only GFAP was used as a marker of mature, reactive astrocytes and use of a secondary astrocyte-specific marker could strengthen these findings. Furthermore, it has been established that exposure to inflammatory cytokines such as IFN $\gamma$, causes astrocytes to become reactive and further skews already-reactive astrocytes to a highly pro-inflammatory phenotype, leading to a greater production of pro-inflammatory cytokines [18] [34] [35]. Conversely, by altering the cytokine profile presented to reactive astrocytes, their reactivity can be lessened. One group demonstrated that administration of IL-10 attenuates astroglial reactivity but has no direct effect on naïve astrocytes [36]. This also correlates with our previous study where there was an increase in IL-10 production in the presence of keratin coatings and IL-4 (M2 control) treatment [24], as well as with the current one where the same keratin and M2 conditioned media yield significantly less reactive (GFAP+) astrocytes and CSPGs (Figure 2 and Figure 3). This was also evidenced by diminished staining (Figures 4(G)-(J) and Figures $5(G)-(J))$. It is important to note that in this study, treatment B, a media solution from the 7 day time point, actually represents media from cells that had been in culture for 7 days, but the accumulation of cell secretion factors was only for 24 hours due to the need to change the media every third day. This may suggest some responses are being underestimated because there may be some factors that are present in lower concentrations than would be expected at a full 7 days of accumulation. Another limitation is that this study is not entirely representative of the in vivo environment and the range of CSPGs generated because various CNS cells produce different CSPGs found in different niches. For example, brevican can be found attached to astrocyte membranes but neurocan and phosphacan are shed into the surrounding milieu and can bind to underlying substrates [15] [37] [38], and neuron-glial antigen 2 (NG2) is predominantly produced by oligodendrocyte progenitors but not astrocytes [39]. Thus, this study indicates the presence of membrane-associated core proteins, aggrecan, and versican, produced by reactive astrocytes but does not take into account those secreted or produced by other glial cells and neurons. It should also be appreciated that different CSPGs have different temporal expression patterns, so future studies should include a range of conditioned media to give a more comprehensive representation of reactive astrocyte response.

It has long been understood that astrocytes respond to cytokines and inflammatory mediators by altering functional and transcriptome profiles [34] [35] [40]. Based on the rapid up-regulation of TGF $\beta$ following CNS injury and its close association with inducing reactive astrogliosis and regulating the ECM, it is also known that treating astrocytes with TGF $\beta$ will cause them to become reactive (i.e. hypertrophy, increased expression of GFAP and ECM proteins) [21] [22] [41]-[43]. This presents a potential problem in vitro because of the presence of serum, an intrinsic source of TGF $\beta$. Some studies have shown that the TGF $\beta$ present in serum exists only in its latent form and thus should have no direct effect on astrocyte reactivity [43] [44]. Nonetheless, this potential confounding effect was considered in the design of the controls for this study. It was effectively shown that the macrophage media alone does not induce reactive astrogliosis (data not shown) and no fluorescence was detected from cells not activated. This discounts most possibilities that the serum may be interfering with the results. It was further shown that not only do astrocytes cultured in RPMI have no statistical difference from the AM naïve astrocyte group, but the macrophage media also does not contribute to changes in reactivity (Figure 1). Additionally, TGF $\beta$ may serve as a measurement of reactive astrogliosis and further work is needed to quantify its concentration within conditioned medias.

Interestingly, we observed a significant decrease in aggrecan production (Figure 1(B)) and GFAP expression (Figure 1(D)) with K-RPMI treatment. The solubilized keratin added to the same macrophage media should indicate if there is any effect due to the possibility of keratin coatings dispersing into the media. From the data it appears keratin may have a direct effect on reactive astrocytes as indicated by the reduction in GFAP and aggrecan production in macrophage media with solubilized keratin (K-RPMI) compared to macrophage media alone (RA-RPMI) (Figure 1).

The role of M1 and M2 macrophages in SCI is becoming a crucial focal point for therapeutic interventions. In this study we demonstrate a functional outcome of macrophages activated in response to a keratin biomaterial. We have previously shown that keratin skews macrophages toward a M2 phenotype significantly greater than a 
collagen biomaterial and promotes an anti-inflammatory cytokine profile [27]. The conditioned media collected from these macrophages reveals a potential new role for keratin in SCI, where it can bias macrophages toward a M2 phenotype which then can down-regulate production of CSPGs and overall reactivity resulting from reactive astrogliosis. In vivo studies will be important to elucidate the functionality of the ensuing reactive astrocytes, but future studies should first evaluate astrocytes that have been cultured with conditioned media for neurite outgrowth potential. While studies on the effects of activated macrophages on reactive astrocytes are ongoing, the reaction of macrophages in response to changes in astrocytes has been demonstrated. One study has shown that viral vector delivery of chABC post-SCI promoted a greater remodeling of CSPGs that was closely associated with CD206+ macrophages, a specific M2 marker [45]. The same study also demonstrated in vitro chABC treatment supported a significant increase in CD206 expression in monocytes. Similarly, macrophages can polarize to opposing phenotypes based on injured neurons, which seem to bias microglia and macrophages to a M1 phenotype [46]. Thus, the glial scar environment is multi-faceted where there is a "vicious cycle" at play that perpetuates the strongly inhibitory milieu.

\section{Conclusion}

Reactive astrogliosis is heavily implicated in the development and propagation of the glial scar forming at the site of SCI. Aside from their physical entanglement of processes, there is a marked up-regulation in CSPGs that further complicate the inhibitory effects on axon outgrowth. The inflammatory response involving the activation of macrophages is known to contribute to this process, where modulating this response to preferentially polarize macrophages to a M2 phenotype has been shown to promote axon regeneration across the lesion site. Here, we establish that a keratin biomaterial that has previously been shown to polarize macrophages to a M2 phenotype can further diminish astrocyte reactivity and CSPG production through conditioned media. Keratin likely has an indirect role, in part, in attenuating reactive astrogliosis through its robust effects on macrophage phenotype, although a direct role cannot be ruled out as keratin in the absence of macrophage conditioning is able to significantly decrease aggrecan and GFAP expression in reactive astrocytes.

\section{Conflict of Interest Statement}

Dr. Mark Van Dyke held stock and was an officer of KeraNetics, LLC, who provided partial funding for this research, at the time the research was conducted. Wake Forest University Health Sciences has a potential financial interest in KeraNetics through a licensing agreement.

\section{Acknowledgements}

The authors would like to thank KeraNetics and the Errett Fisher Foundation for funding support.

\section{References}

[1] Fawcett, J.W. and Asher, R.A. (1999) The Glial Scar and Central Nervous System Repair. Brain Research Bulletin, 49, 377-391. http://dx.doi.org/10.1016/S0361-9230(99)00072-6

[2] Reier, P.J.S. and Guth, L. (1983) The Astrocytic Scar as an Impediment to Regeneration in the Central Nervous System. In: Kao, R.P. and Reier, P.J., Ed., Spinal Cord Reconstruction, Raven Press, New York, 163-195.

[3] Silver, J. and Miller, J.H. (2004) Regeneration beyond the Glial Scar. Nature Reviews Neuroscience, 5, 146-156. http://dx.doi.org/10.1038/nrn1326

[4] Faulkner, J.R., et al. (2004) Reactive Astrocytes Protect Tissue and Preserve Function after Spinal Cord Injury. Journal of Neuroscience, 24, 2143-2155. http://dx.doi.org/10.1523/JNEUROSCI.3547-03.2004

[5] Sofroniew, M.W. (2009) Molecular Dissection of Reactive Astrogliosis and Glial Scar Formation. Trends in Neurosciences, 32, 638-647. http://dx.doi.org/10.1016/j.tins.2009.08.002

[6] Bradbury, E.J. and Carter, L.M. (2011) Manipulating the Glial Scar: Chondroitinase ABC as a Therapy for Spinal Cord Injury. Brain Research Bulletin, 84, 306-316. http://dx.doi.org/10.1016/j.brainresbull.2010.06.015

[7] Gu, W.L., et al. (2009) Chondroitin Sulfate Proteoglycans Regulate the Growth, Differentiation and Migration of Multipotent Neural Precursor Cells through the Integrin Signaling Pathway. BMC Neuroscience, 10, 128. http://dx.doi.org/10.1186/1471-2202-10-128

[8] Landolt, R.M., et al. (1995) Versican Is Selectively Expressed in Embryonic-Tissues That Act as Barriers to Neural 
Crest Cell-Migration and Axon Outgrowth. Development, 121, 2303-2312.

[9] Treloar, H.B., Nurcombe, V. and Key, B. (1996) Expression of Extracellular Matrix Molecules in the Embryonic Rat Olfactory Pathway. Journal of Neurobiology, 31, 41-55. http://dx.doi.org/10.1002/(SICI)1097-4695(199609)31:1<41::AID-NEU4>3.0.CO;2-F

[10] Brakebusch, C., et al. (2002) Brevican-Deficient Mice Display Impaired Hippocampal CA1 Long-Term Potentiation But Show No Obvious Deficits in Learning and Memory. Molecular and Cellular Biology, 22, 7417-7427. http://dx.doi.org/10.1128/MCB.22.21.7417-7427.2002

[11] Pizzorusso, T., Medini, P., Berardi, N., et al. (2002) Reactivation of Ocular Dominance Plasticity in the Adult Visual Cortex. Science, 298, 1248-1251. http://dx.doi.org/10.1126/science.1072699

[12] Davies, S.J.A., Fitch, M.T., Memberg, S.P., et al. (1997) Regeneration of Adult Axons in White Matter Tracts of the Central Nervous System. Nature, 390, 680-683.

[13] McKeon, R.J., Jurynec, M.J. and Buck, C.R. (1999) The Chondroitin Sulfate Proteoglycans Neurocan and Phosphacan Are Expressed by Reactive Astrocytes in the Chronic CNS Glial Scar. Journal of Neuroscience, 19, 10778-10788.

[14] Afshari, F.T., Kwok, J.C., White, L., et al. (2010) Schwann Cell Migration Is Integrin-Dependent and Inhibited by Astrocyte-Produced Aggrecan. Glia, 58, 857-869. http://dx.doi.org/10.1002/glia.20970

[15] Asher, R.A., Morgenstern, D.A., Fidler, P.S., et al. (2000) Neurocan Is Upregulated in Injured Brain and in Cytokine-Treated Astrocytes. The Journal of Neuroscience, 20, 2427-2438.

[16] Fidler, P.S., Schuette, K., Asher, R.A., et al. (1999) Comparing Astrocytic Cell Lines That Are Inhibitory or Permissive for Axon Growth: The Major Axon-Inhibitory Proteoglycan Is NG2. The Journal of Neuroscience, 19, 8778-8788.

[17] Rhodes, K.E. and Fawcett, J.W. (2004) Chondroitin Sulphate Proteoglycans: Preventing Plasticity or Protecting the CNS? Journal of Anatomy, 204, 33-48. http://dx.doi.org/10.1111/j.1469-7580.2004.00261.x

[18] Sofroniew, M.V. (2014) Multiple Roles for Astrocytes as Effectors of Cytokines and Inflammatory Mediators. The Neuroscientist, 20, 160-172. http://dx.doi.org/10.1177/1073858413504466

[19] Kigerl, K.A., Gensel, J.C., Ankeny, D.P., et al. (2009) Identification of Two Distinct Macrophage Subsets with Divergent Effects Causing Either Neurotoxicity or Regeneration in the Injured Mouse Spinal Cord. The Journal of Neuroscience, 29, 13435-13444. http://dx.doi.org/10.1523/JNEUROSCI.3257-09.2009

[20] Flanders, K.C., Ren, R.F. and Lippa, C.F. (1998) Transforming Growth Factor-Betas in Neurodegenerative Disease. Progress in Neurobiology, 54, 71-85. http://dx.doi.org/10.1016/S0301-0082(97)00066-X

[21] Smith, G.M. and Strunz, C. (2005) Growth Factor and Cytokine Regulation of Chondroitin Sulfate Proteoglycans by Astrocytes. Glia, 52, 209-218. http://dx.doi.org/10.1002/glia.20236

[22] Ricardo, S.D., van Goor, H. and Eddy, A.A. (2008) Macrophage Diversity in Renal Injury and Repair. Journal of Clinical Investigation, 118, 3522-3530. http://dx.doi.org/10.1172/JCI36150

[23] Fearing, B.V., Hartley, C., Dayton, O., Sherwood, G., Aboushwareb, T. and Van Dyke, M.E. (2014) Treatment of a Spinal Cord Hemi-Transection Injury with Keratin Biomaterial Hydrogel Elicits Recovery and Tissue Repair. ISRN Biomaterials, 2014, 1-9.

[24] Fearing, B.V. and Van Dyke, M.E. (2014) In Vitro Response of Macrophage Polarization to a Keratin Biomaterial. Acta Biomaterialia, 10, 3136-3144. http://dx.doi.org/10.1016/j.actbio.2014.04.003

[25] de Guzman, R.C., Merrill, M.R., Richter, J.R., et al. (2011) Mechanical and Biological Properties of Keratose Biomaterials. Biomaterials, 32, 8205- 8217. http://dx.doi.org/10.1016/j.biomaterials.2011.07.054

[26] Sierpinski, P., Garrett, J., Ma, J., et al. (2008) The Use of Keratin Biomaterials Derived from Human Hair for the Promotion of Rapid Regeneration of Peripheral Nerves. Biomaterials, 29, 118-128. http://dx.doi.org/10.1016/j.biomaterials.2007.08.023

[27] Yu, P., Wang, H., Katagiri, Y. and Geller, H.M. (2012) An in Vitro Model of Reactive Astrogliosis and Its Effect on Neuronal Growth. Methods in Molecular Biology, 814, 327-340. http://dx.doi.org/10.1007/978-1-61779-452-0_21

[28] Bradbury, E.J., Moon, L.D., Popat, R.J., et al. (2002) Chondroitinase ABC Promotes Functional Recovery after Spinal Cord Injury. Nature, 416, 636-640. http://dx.doi.org/10.1038/416636a

[29] Mckeon, R.J., Hoke, A. and Silver, J. (1995) Injury-Induced Proteoglycans Inhibit the Potential for Laminin-Mediated Axon Growth on Astrocytic Scars. Experimental Neurology, 136, 32-43. http://dx.doi.org/10.1006/exnr.1995.1081

[30] Lemons, M.L., Sandy, J.D., Anderson, D.K., et al. (2003) Intact Aggrecan and Chondroitin Sulfate-Depleted Aggrecan Core Glycoprotein Inhibit Axon Growth in the Adult Rat Spinal Cord. Experimental Neurology, 184, 981-990. http://dx.doi.org/10.1016/S0014-4886(03)00383-2

[31] Steele, M.L. and Robinson, S.R. (2012) Reactive Astrocytes Give Neurons Less Support: Implications for Alzheimer's Disease. Neurobiology of Aging, 33, 423.e1-423.13. http://dx.doi.org/10.1016/j.neurobiolaging.2010.09.018 
[32] Haan, N., Zhu, B., Wang, J., Wei, X. and Song, B. (2015) Crosstalk between Macrophages and Astrocytes Affects Proliferation, Reactive Phenotype and Inflammatory Response, Suggesting a Role during Reactive Gliosis Following Spinal Cord Injury. Journal of Neuroinflammation, 12, 109. http://dx.doi.org/10.1186/s12974-015-0327-3

[33] Apelt, J. and Schliebs, R. (2001) Beta-Amyloid-Induced Glial Expression of Both Pro- and Anti-Inflammatory Cytokines in Cerebral Cortex of Aged Transgenic Tg2576 Mice with Alzheimer Plaque Pathology. Brain Research, 894, 21-30. http://dx.doi.org/10.1016/S0006-8993(00)03176-0

[34] Hamby, M.E., Coppola, G., Ao, Y., et al. (2012) Inflammatory Mediators Alter the Astrocyte Transcriptome and Calcium Signaling Elicited by Multiple G-Protein-Coupled Receptors. Journal of Neuroscience, 32, 14489-14510. http://dx.doi.org/10.1523/JNEUROSCI.1256-12.2012

[35] Zamanian, J.L., Xu, L., Foo, L.C., et al. (2012) Genomic Analysis of Reactive Astrogliosis. Journal of Neuroscience, 32, 6391-6410. http://dx.doi.org/10.1523/JNEUROSCI.6221-11.2012

[36] Balasingam, V. and Yong, V.W. (1996) Attenuation of Astroglial Reactivity by Interleukin-10. Journal of Neuroscience, 16, 2945-2955.

[37] Dow, K.E. and Wang, W. (1998) Cell Biology of Astrocyte Proteoglycans. Cellular and Molecular Life Sciences, 54, 567-581. http://dx.doi.org/10.1007/s000180050185

[38] Yamada, H., Fredette, B., Shitara, K., et al. (1997) The Brain Chondroitin Sulfate Proteoglycan Brevican Associates with Astrocytes Ensheathing Cerebellar Glomeruli and Inhibits Neurite Outgrowth from Granule Neurons. The Journal of Neuroscience, 17, 7784-7795.

[39] Jones, L.L., Yamaguchi, Y., Stallcup, W.B., et al. (2002) NG2 Is a Major Chondroitin Sulfate Proteoglycan Produced after Spinal Cord Injury and Is Expressed by Macrophages and Oligodendrocyte Progenitors. The Journal of Neuroscience, 22, 2792-2803

[40] Eddleston, M. and Mucke, L. (1993) Molecular Profile of Reactive Astrocytes-Implications for Their Role in Neurologic Disease. Neuroscience, 54, 15-36. http://dx.doi.org/10.1016/0306-4522(93)90380-X

[41] Hsiao, T.W., Swarup, V.P., Kuberan, B., et al. (2013) Astrocytes Specifically Remove Surface-Adsorbed Fibrinogen and Locally Express Chondroitin Sulfate Proteoglycans. Acta Biomaterialia, 9, 7200-7208. http://dx.doi.org/10.1016/j.actbio.2013.02.047

[42] Yu, P.W., Katagiri, Y. and Geller, H., Eds. (2012) An in Vitro Model of Reactive Astrogliosis and Its Effect on Neuronal Growth. In: Milner, R., Ed., Astrocytes: Methods and Protocols, Volume 814, Springer Science + Business LLC, New York, 327-340.

[43] Khan, S.A., Joyce, J. and Tsuda, T. (2012) Quantification of Active and Total Transforming Growth Factor-Beta Levels in Serum and Solid Organ Tissues by Bioassay. BMC Research Notes, 5, 636. http://dx.doi.org/10.1186/1756-0500-5-636

[44] Oida, T. and Weiner, H.L. (2010) Depletion of TGF-Beta from Fetal Bovine Serum. Journal of Immunological Methods, 362, 195-198. http://dx.doi.org/10.1016/j.jim.2010.09.008

[45] Bartus, K., James, N.D., Didangelos, A., et al. (2014) Large-Scale Chondroitin Sulfate Proteoglycan Digestion with Chondroitinase Gene Therapy Leads to Reduced Pathology and Modulates Macrophage Phenotype following Spinal Cord Contusion Injury. The Journal of Neuroscience, 34, 4822-4836. http://dx.doi.org/10.1523/JNEUROSCI.4369-13.2014

[46] Huang, Y.C. and Feng, Z.P. (2013) The Good and Bad of Microglia/Macrophages: New Hope in Stroke Therapeutics. Acta Pharmacologica Sinica, 34, 6-7. http://dx.doi.org/10.1038/aps.2012.178 


\section{Acronyms}

$\begin{array}{lc}\text { AM } & \text { Astrocyte Media } \\ \text { ANOVA } & \text { Analysis of Variance } \\ \text { ATCC } & \text { American Type Culture Collection } \\ \text { BSA } & \text { Bovine Serum Albumin } \\ \text { BSB } & \text { Blood Spinal Cord Barrier } \\ \text { chABC } & \text { Chondroitinase ABC } \\ \text { CNS } & \text { Central Nervous System } \\ \text { CS } & \text { Chondroiting Sulfate } \\ \text { CSPGs } & \text { Chondroitin Sulfate Proteoglycans } \\ \text { DI } & \text { Deionized } \\ \text { ECM } & \text { Extracellular Matrix } \\ \text { FITC } & \text { Fluorescein Isothiocyanate } \\ \text { GAG } & \text { Glucosaminoglycan } \\ \text { GFAP } & \text { Glial Fibrillary Acidic Protein } \\ \text { Gy } & \text { Gray } \\ \text { HEPES } & \text { 4-(2-Hydroxyethyl)-1-piperazineethanesulfonic acid } \\ \text { IL } & \text { Interleukin } \\ \text { INF } & \text { Interferon } \\ \text { K-MCM } & \text { Keratin-Treated Macrophage Conditioned Media } \\ \text { LPS } & \text { Lipopolysaccharide } \\ \text { MCM } & \text { Macrophage Conditioned Media } \\ \text { NaOH } & \text { Sodium Hydroxide } \\ \text { NG2 } & \text { Neuron-Glial antigen 2 } \\ \text { NIH } & \text { National Institutes of Health } \\ \text { PBS } & \text { Phosphate Buffered Saline } \\ \text { PFA } & \text { Paraformaldehyde } \\ \text { PMA } & \text { PhorbolMyristate Acetate } \\ \text { RA } & \text { Reactive Astrocytes } \\ \text { RA-AM } & \text { Reactive Astrocytes-Astrocyte Media } \\ \text { RFU } & \text { Relative Fluorescence Units } \\ \text { RPMI } & \text { Roswell Park Memorial Institute } \\ \text { RT } & \text { Room Temperature } \\ \text { SCI } & \text { Spinal Cord Injury } \\ \text { SEM } & \text { Standard Error of the Mean } \\ \text { TDM } & \text { THP-1 Derived Macrophages } \\ \text { TGF } & \text { Transforming Growth Factor } \\ \text { TNF } & \text { Tumor Necrosis Factor } \\ \text { Tris } & \text { Tris(hydroxymethyl)aminomethane } \\ & \end{array}$

